\title{
Near-infrared holographic photorefractive recording under applied electric field in undoped $\mathrm{Bi}_{12} \mathrm{TiO}_{20}$ sillenite crystal
}

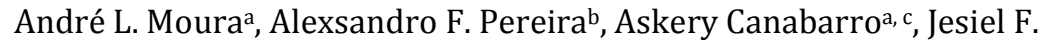 \\ Carvalho $^{d}$, Ivan de Oliveirae, Pedro V. dos Santos ${ }^{\mathrm{b}, 1}$ \\ aGrupo de Física da Matéria Condensada, Núcleo de Ciências Exatas, Campus Arapiraca,Universidade Federal de Alagoas, 57309-005, \\ Arapiraca-AL, Brazil \\ bInstituto de Física, Universidade Federal de Alagoas, 57072-970, Maceió-AL, Brazil \\ 'International Institute of Physics, Federal University of Rio Grande do Norte, 59070-405,Natal, Brazil \\ 'Instituto de Física Universidade Federal de Goiás, Goiânia, GO, Brazil \\ ${ }^{\mathrm{e}}$ GOMNI-Grupo de Optica e Modelagem Numérica, Faculdade de Tecnologia/UNICAMP, Limeira, SP, Brazil
}

\begin{abstract}
Direct holographic recording in undoped $\mathrm{Bi}_{12} \mathrm{TiO}_{20}$ crystal at $1064 \mathrm{~nm}$ is investigated aiming the characterization of diffraction efficiency under action of applied dc electric field $\left(E_{0}\right)$. An enhancement of 12 -fold in the diffraction efficiency was revealed when $E_{0}$ increased from 0.0 to $4.2 \mathrm{kV} / \mathrm{cm}$. The theoretical dependence of the diffraction efficiency upon $E_{0}$ was investigated using the standard model for photorefractivity and the results showed a good experimental data fitting, allowing the computation of the effective trap concentration $\left(N_{D}\right)_{\text {eff }} \approx 5.3 \times 10^{15} \mathrm{~cm}^{-3}$ which is responsible by the recording mechanism into the BTO crystal sample. The type of charge carrier involved in the recording mechanism in the nearinfrared region is also discussed.
\end{abstract}

Keywords: Diffraction, holographic gratings, holographic recording, photorefractive effect, photorefractive materials.

\section{Introduction}

Photonics requires the characterization of optical properties of a myriad of materials, such as glasses, crystals, and nanocrystal powders. Amidst the crystalline ones, the photorefractives are interesting for hologram recording due to the photoconductive and electro-optic effects which allow registering a pattern of light as a modulation of the refractive index [1]. Sillenites $\left(\mathrm{Bi}_{12} \mathrm{MO}_{20}\right.$ with $\mathrm{M}=\mathrm{Si}$, Ge or Ti) have been extensively characterized in the visible spectral range. Regarding longer wavelengths, the $\mathrm{Bi}_{12} \mathrm{TiO}_{20}$ (BTO) crystal allows holographic recording in the near infrared region of the electromagnetic spectrum where both optical activity and optical absorption are lower than in the visible region [2, 3], and presents high effective electro-optic coefficient $(\approx 5.5 \mathrm{pm} / \mathrm{V})[4]$.

In [2] and [3] the authors investigated stationary (nonmoving) photorefractive hologram recorded in undoped BTO crystal at $1064 \mathrm{~nm}$ and $780 \mathrm{~nm}$, respectively. These two experiments were carried out with and without a mechanism of pre-exposure to light, but no one external electric field was applied to the

\footnotetext{
${ }^{1}$ Corresponding author

Email address: pedro@fis.ufal.br (Pedro V.dos Santos)
} 
samples, and in both experiments the results revealed recorded holographic grating very weak in spite of the diffraction efficiency measured with pre-exposure was approximately 3 times greater than that without pre-exposure. Another way to improve the recording characteristics at $1064 \mathrm{~nm}$ or $780 \mathrm{~nm}$ is by applying an ac or dc electric field to the sample, but there are not experimental results in the literature that report this fact for photorefractive recording in undoped BTO at those wavelengths to the best of our knowledge. In fact, it is well known that photorefractive holograms produced under the action of externally applied electric field $\left(E_{0}\right)$ exhibit comparatively large diffraction efficiencies [5, 6]. However, the action of the $E_{0}$ perturbs the crystal index ellipsoid due to the electro-optic effect, inducing a birefringence in the medium making the diffracted beams elliptically polarized; this phenomena produces additional difficulties to separate the diffracted beams from the signal and pump ones and consequently making difficult to determine the diffraction efficiency and also to improve the signal-to-noise ratio (SNR) by simply doing the use of a polarization analyzer $[3,5,7]$. In order to overcome this difficult some phase modulation techniques that are applied to one of the recording beams have been proposed $[8,9,10,11]$. In this work we take advantage of phase-sensitive detection and measure diffraction efficiency by direct chopping of elliptically polarized diffracted beams in photorefractive hologram recorded in undoped BTO crystal at $1064 \mathrm{~nm}$ under the action of a dc $E_{0}$. Here the use of the phase-sensitive technique permitted to detect at ease the very weak direct recording at $1064 \mathrm{~nm}$ without externally applied electric field in an undoped $\mathrm{Bi}_{12} \mathrm{TiO}_{20}$ sillenite. The effects of $E_{0}$ on the diffraction efficiency were both experimentally and theoretically investigated and the results permitted to compute the effective trap concentration $\left(N_{D}\right)_{\text {eff }}$ in a way that it is not necessary to vary the angle between the recording beams.

\section{Experiment}

Holograms were recorded in an undoped BTO sample using $\lambda=1064 \mathrm{~nm}$ beams from a $50 \mathrm{~mW}$ solid state laser in the interferometric arrangement shownin Fig. 1. The intensities of the recording beams measured on the input face of the sample corresponded to a total intensity of $0.45 \mathrm{~W} / \mathrm{cm}^{2}$, with the signal beam intensity being $1 / 36$ from the pump beam intensity. The diameter of the unexpanded beam is $2 \mathrm{~mm}$.

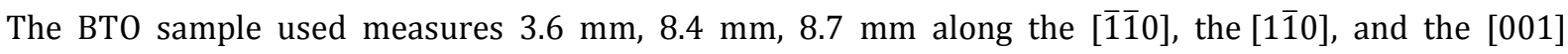
directions, respectively. Thelight interference pattern produced by the two beams (with the electrical field vector being perpendicular to the plane of incidence) was always projected onto the ( $\overline{1} \overline{1} 0)$-crystal face as depicted in Fig. 2, with the [001]-crystal axis being perpendicular to the incidence plane and to the recorded grating vector $\mathbf{K}$, with $|\mathbf{K}|=2 \pi / \Lambda$, where $\Lambda=(\lambda / 2) \sin \theta$ is the spatial frequency of grating, $\lambda$ is the laser wavelength, and $2 \theta$ is the angle between the recording beams. In order to ensure similar initial conditions for the sample, prior to each experimental run it was always illuminated for 25 minutes with one $\left(I_{S}{ }^{0}\right.$ : signal beam intensity) of the two $1064 \mathrm{~nm}$ recording beams while the other one $\left(I_{R}{ }^{0}:\right.$ pump beam intensity) remained blocked by the shutter $\mathrm{SH}_{1}$. In this experiment the shutters were adjusted to run in opposite modes, i.e., when $\mathrm{SH}_{1}$ is closed (opened) $\mathrm{SH}_{2}$ is simultaneously opened (closed). After that time of 25 minutes, $\mathrm{SH}_{1}$ is opened and the light interference pattern writes a photorefractive hologram in the sample producing diffracted beams $\left(I_{d}\right)$ in the $I_{S}$ and $I_{R}$ directions. 


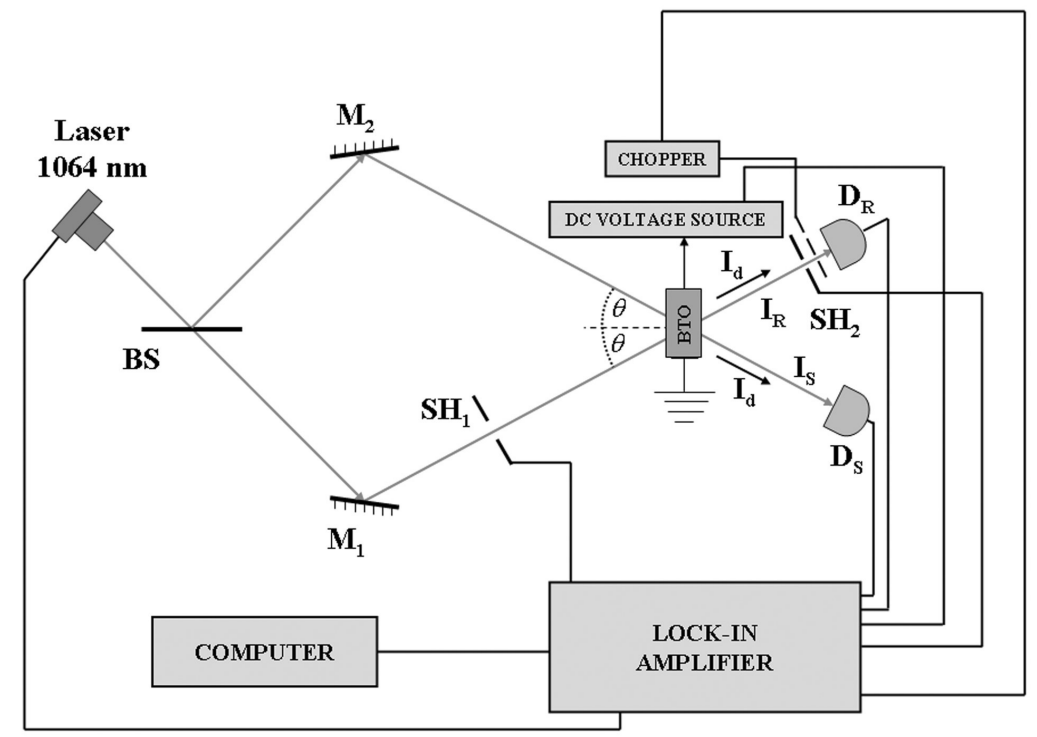

Figure 1: Holographic arrangement: BS is beam-splitter; $\mathrm{M}_{1}$ and $\mathrm{M}_{2}$ are mirrors; $\mathrm{SH}_{1}$ and $\mathrm{SH}_{2}$ are shutters; $I_{R}, I_{S}$ and $I_{d}$, are, respectively, the pump, signal and diffracted beams at the BTO crystal output; $D_{R}$ and $D_{S}$ photodetectors.

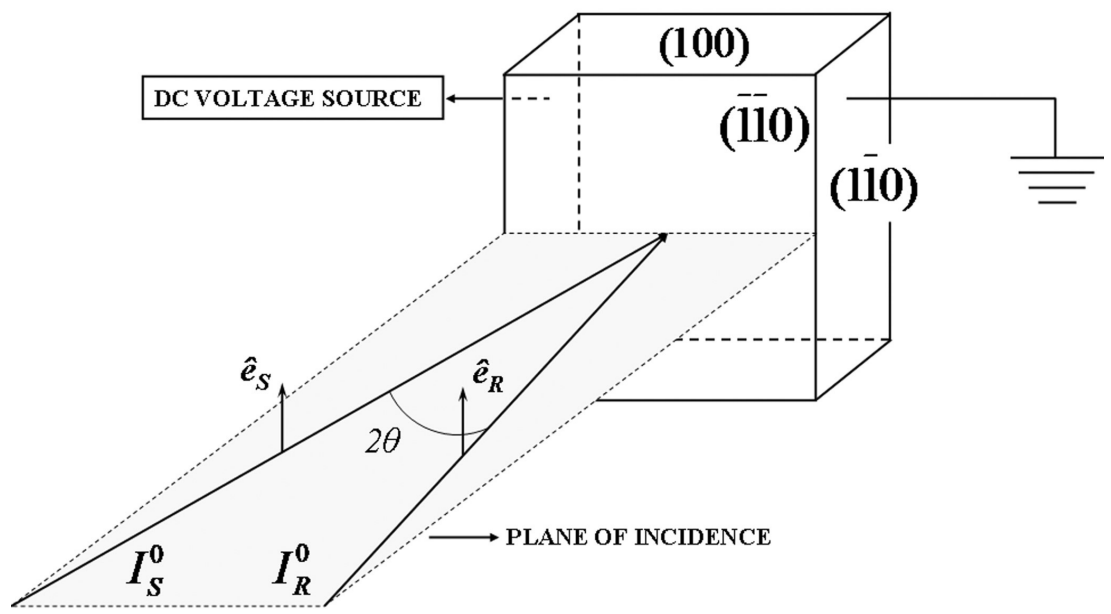

Figure 2: BTO crystal orientation. Intensities $\left(I_{S}^{0}\right.$ and $\left.I_{R}^{0}\right)$ and polarizations $\left(\hat{e}_{S}\right.$ and $\left.\hat{e}_{R}\right)$ of the recording beams at the input face of the sample.

The intensity $I_{S}\left(I_{R}\right)$ plus the diffracted signal in its direction are measured by thephotodetector $D_{S}\left(D_{R}\right)$. When the shutter $\mathrm{SH}_{1}$ is closed again ( $\mathrm{SH}_{2}$ is opened) the sample is illuminated uniformly by the signal beam, causing the erasure of the hologram and consequently producing an in-Bragg diffracted beam which is chopped at $1 \mathrm{kHz}$ and measured by the photodetector $D_{R}$. The photocurrents generated in $D_{S}$ and $D_{R}$ are fed into the lock-in amplifier connected to a computer. Calibration curves of the light intensities measured before $D_{S}$ and $D_{R}$ versus the corresponding lock-in voltages were prepared in order to directly compute the absolute value of the diffraction efficiency as $\eta=I_{d} /\left(I_{d}+I_{S}\right)$ and measured at the beginning of 
erasure (bulk absorption and interface losses are neglected). An external dc voltage is applied between the silver electrodes painted on the side surfaces of the sample, $(1 \overline{1} 0)$ and $(\overline{1} 10)$ faces, making the resultant external electric field always perpendicular to the [001]-crystal axis. The spatial frequency of the recorded grating is $\Lambda=1.55 \mu \mathrm{m}$. As can be seen the setup presented here is identical to those normally used to record holograms in a two-wave mixing (TWM) configuration. The difference here is the lock-in amplifier used to detect the chopped weak diffracted signal behind the sample, and also to control others parts of the experiment as the shutters and the dc voltage source.

\section{Results and discussion}

After holographic recording for 5 minutes using the setup shown in Fig. 1, the reference beam is blocked by $\mathrm{SH}_{1}$ and the signal one is used at the same time to uniformly erase the hologram and as an in- Bragg probe beam to measure the time evolution of diffraction efficiency $\eta$. The time evolution of the chopped diffracted beam intensity was measured at the BTO sample output for different amplitudes of $E_{0}$ and is shown in Fig. 3. Clearly is observed an enhancement in the diffracted signal when the amplitude of $E_{0}$ is increased. This enhancement is attributed to the increase of the photorefractive response of the BTO crystal to $E_{0}[5,6]$. It is important to point out here that measurements of diffraction efficiencies were also carried out for different chopping frequencies of $200 \mathrm{~Hz}$, and $2 \mathrm{kHz}$, and no modification in the time evolution of the diffraction efficiency was observed (results not shown here). An analysis of the time decay curves depicted in Fig. 3 showed that they can be well fitted with a single exponential function indicating the probable participation of a unique photoactive center [12] responsible for holographic recording. The dependence of the diffraction efficiency upon $E_{0}$ was examined and the result is depicted (symbols) in Fig. 4. As can inferred from the experimental data, the diffraction efficiency increased by a factor of 12 -fold when the amplitude of the $E_{0}$ increased from 0 to $4.2 \mathrm{kV} / \mathrm{cm}$.

Some discussion is necessary to explain our results about the mechanism involved in the process of the direct photorefractive recording in the BTO sample using infrared laser at $1064 \mathrm{~nm}$ (Energy $h v \approx 1.2 \mathrm{eV}$ ). The literature presents detailed studies about the structure of localized states within the band-gap of the undoped BTO crystal. These states have activation energies ranging from 0.1 to $3.0 \mathrm{eV}$ and they are reported in a more complete view in references $[2,12,13]$. Because of the recording photon energy at 1.2 $\mathrm{eV}$ we have utilized a simplified energy diagram shown in Figure 5 exhibiting only the relevant photoactive centers of the BTO to explain our approaches. Figure 5 shows a possible recording mechanism (mechanism 1) in which one deep trap (DT) center is placed $1.3 \mathrm{eV}$ below the conduction band (CB). In this mechanism the DT level can work as an electron-donor center from which electrons can be excited by the recording photons to reach the $\mathrm{CB}$, even missing $\Delta U=0.1 \mathrm{eV}$ to complete the excitation gap. In the conduction band these electrons can move by diffusion or drift (when $E_{0}$ is applied) for some distance and then recombine in DT and so permitting the building of the photorefractive grating. In another similar mechanism (mechanism 2) the D level could be thought running as a hole-donor center from which holes would be excited by recording photons to the valence band, however this phenomenon has been reported as an unlikely process $[2,3]$ in sillenite since the $D$ level is filled with electrons so that the concentration of holes does not exist or it is very low for to produce a detectable hole-based photorefractive grating. So we believe that the direct photorefractive recording in undoped BTO crystal using infrared laser at $1064 \mathrm{~nm}$ 
is essentially dominated by electron-type charge carrier and is recorded in the localized state at around $1.3 \mathrm{eV}$. This approach corroborates the results of the diffraction efficiency measurements as a function of time shown in figure 3 in which the adjustments with a single exponential indicates the participation of only one photoactive center in the process of photorefractive recording.

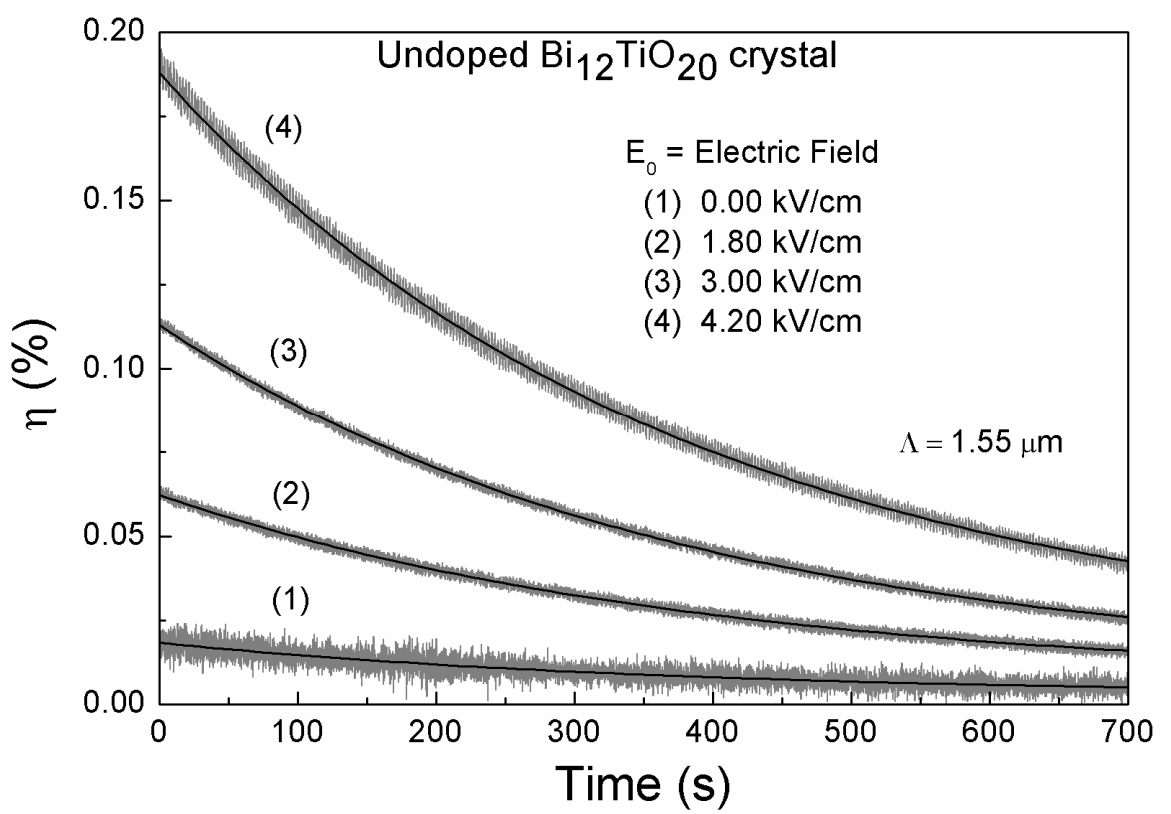

Figure 3: Diffraction efficiency as a function of time during the hologram erasure for different applied electric field. Gray lines are the experimental results and the tick lines are adjustments with a single exponential function.

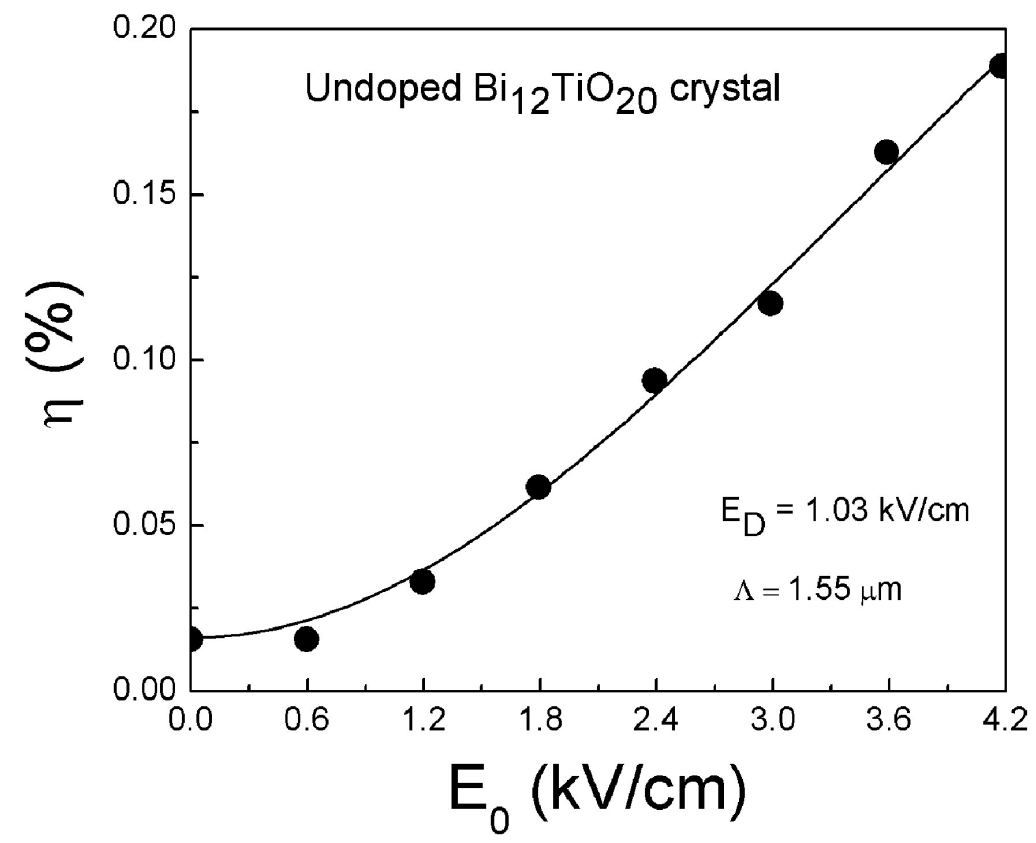

Figure 4: Diffraction efficiency as a function of the applied electric field. The solid line represents the theoretical fit obtained from Eq. 4 adjusted to the experimental data (symbols). 


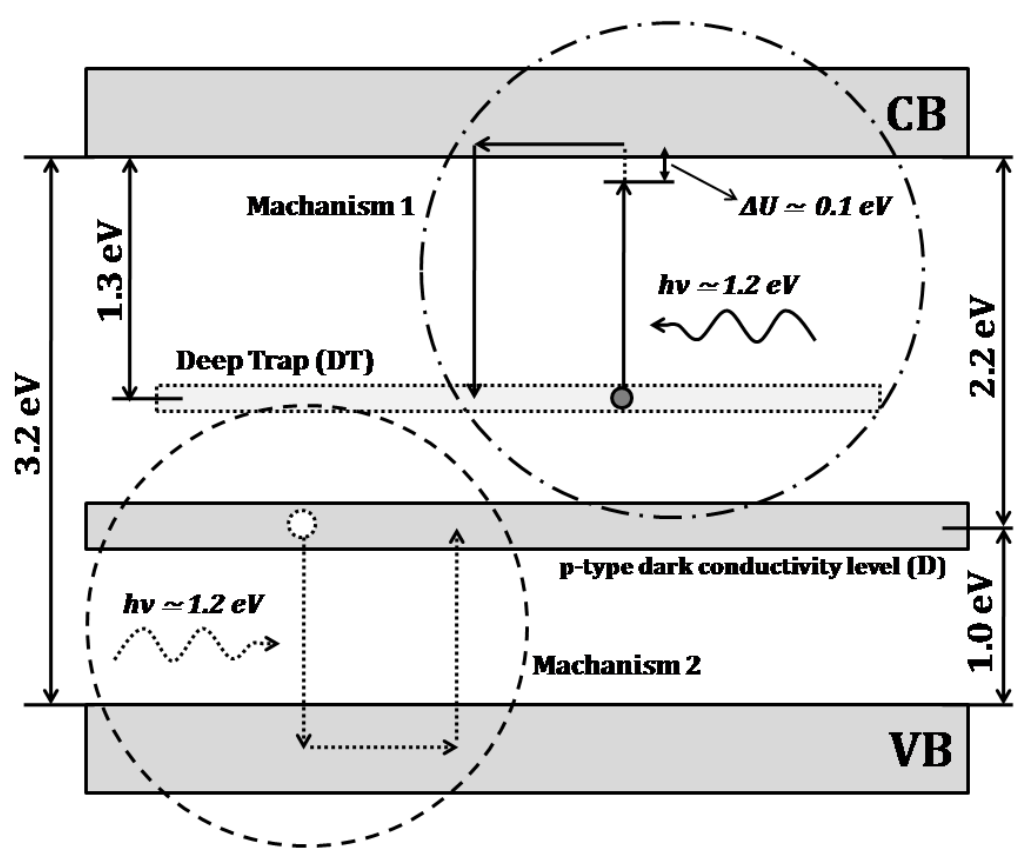

Figure 5: Simplified energy diagram illustrating the possible ways for direct nearinfrared photorefractive recording at $1064 \mathrm{~nm}$. Mechanism 1 is the most likely and highlights the deep trap (DT) level at $1.3 \mathrm{eV}$ below the conduction band (CB) as an electron-donor photoactive center responsible by recording at $1064 \mathrm{~nm}$. Mechanism 2 shows the donor (D) level at $1.0 \mathrm{eV}$ above the valence band (VB) as a possible hole-donor center, but it is an unlikely process $[2,3]$ in sillenite.

The enhancement of the diffraction efficiency as a function of $E_{0}$ was theoretically investigated by using the standard model $[12,14]$ and taking into account that only one photoactive center is participating in the photorefractive recording and erasure processes.

A recorded photorefractive hologram (or diffraction grating) may be read by a beam which is Braggmatched to the grating and this beam is scattered with diffraction efficiency (for $\eta<<1)[12,14]$

$$
\eta \propto\left|E_{s c}\right|^{2}
$$

where $E_{s c}$ is the space-charge-field $[12,14]$ given by:

$$
E_{S C}=i m \frac{E_{D}+i E_{0}}{1+\frac{E_{D}+i E_{0}}{E_{q}}}
$$

with $m$ being the visibility of the fringes of interference, $E_{D}=2 \pi k_{B} T / e \Lambda$, and $E_{q}=e \Lambda\left(N_{D}\right)_{\text {eff }} /\left(2 \pi \varepsilon \varepsilon_{0}\right)$, where $k_{B}$ is the Boltzmann constant, $T$ the absolute temperature, $e$ the elementary charge, $\left(N_{D}\right)_{e f f}$ the effective trap or effective donor center concentration responsible to recording the photorefractive grating, $\varepsilon$ the dielectric constant, and $\varepsilon_{0}$ is the dielectric permittivity of vacuum. Equation 2 as showed is valid for spacecharge-field produced by hole-type charge carrier. If the charge carrier is electron the field $E_{D}$ should be replaced by - $E_{D}$ and $E_{q}$ by $-E_{q}$ in Equation 2. It is straightforward, regardless of whether the charge carrier is hole or electron, to obtain that

$$
\eta \propto m^{2} \frac{E_{0}^{2}+E_{D}^{2}}{\left(E_{0} / E_{q}\right)^{2}+\left[\left(E_{q}+E_{D}\right) / E_{q}\right]^{2}}
$$


In this approach the accumulative effect of charges in the shadow regions near the side surfaces electrodes $[15,16]$ was not take in account. Such effect contributes to decrease (screen) the action of the $E_{0}$ in the bulk of the material $[15,16]$. In order to take into account the effect of screening produced by that charge distribution, we introduced a parameter $\xi$, called field factor, which can vary from 0 (no applied electric field) to 1 (no screening effect) and the Eq. 3 can now be written as

$$
\eta \propto m^{2} \frac{\left(\xi E_{0}\right)^{2}+E_{D}^{2}}{\left(\xi E_{0} / E_{q}\right)^{2}+\left[\left(E_{q}+E_{D}\right) / E_{q}\right]^{2}} .
$$

Equation 4 was fitted to the experimental data and the result is indicated by the solid line in plot of Fig. 4, showing a good agreement between the theoretical curve and experimental data. Using $E_{D}=1.03 \mathrm{kV} / \mathrm{cm}(T$ $=295 \mathrm{~K}$ ) we find $\xi \simeq 1$ and $E_{q} \simeq 5.0 \mathrm{kV} / \mathrm{cm}$. Using this value of $E_{q}$ and $\varepsilon=47$ [17] we computed an acceptor concentration in the crystal of $\left(N_{D}\right)_{\text {eff }} \simeq 5.3 \times 10^{15} \mathrm{~cm}^{-3}$. This value is of the same order of magnitude than that one obtained by Odoulov et al. at $1064 \mathrm{~nm}$ [2], but that was estimated by using the technique proposed by Klein and Valley [18] which has an experimental difficulty that is the necessity to vary the angle $2 \theta$ between the recording beams in order to indirectly compute theacceptor concentration. In our approach presented here that difficulty does not exist since the angle $2 \theta$ remains fixed. The field factor value $\xi \simeq 1$ indicates that the influence of the screening effect is very weak and it seems not to be a limiting factor of the enhancement of the diffraction efficiency as a function of $E_{0}$ in the recording region at $1064 \mathrm{~nm}$.

\section{Conclusion}

We measured diffraction efficiency by direct chopping of elliptically polarized diffracted beams in photorefractive holographic grating recorded in undoped $\mathrm{Bi}_{12} \mathrm{TiO}_{20}$ crystal at $1064 \mathrm{~nm}$ under action of an applied dc electric field $\left(E_{0}\right)$ using a two-wave mixing configuration. The experimental results showed a 12 -fold enhancement in the diffraction efficiency when $E_{0}$ was ranged from 0 to $4.2 \mathrm{kV} / \mathrm{cm}$. This enhancement was attributed to photorefractive response of the material to $E_{0}$ and was investigated theoretically using the standard model for photorefractivity and the results showed to agree quite well with experimental data allowing to compute the effective electron-donor concentration $\left(N_{D}\right)_{\text {eff }}$ responsible for direct photorefractive recording in $\mathrm{Bi}_{12} \mathrm{TiO}_{20}$ crystal at $1064 \mathrm{~nm}$.

\section{Funding}

Conselho Nacional de Desenvolvimento Científico e Tecnológico (CNPq); Financiadora de Estudos e Projetos (FINEP); Coordenação de Aperfeiçoamento de Pessoal de Nível Superior (CAPES); Fundação de Amparo à Pesquisa do Estado de Alagoas (FAPEAL).

\section{Acknowledgements}

We acknowledge financial support from the Brazilian Agencies: CNPq, FINEP, CAPES, and FAPEAL. AC acknowledges the John Templeton Foundation via the Grant Q-CAUSAL No. 61084, the Serrapilheira Institute (Grant No. Serra-1708-15763), the Brazilian National Council for Scientific and Technological Development (CNPq) via the National Institute for Science 
and Technology on Quantum Information (INCT-IQ) and Grant № 423713/2016-7. AC also acknowledges UFAL for a paid license for scientific cooperation at UFRN.

\section{References}

[1] P. Günter, J. Huignard, Photorefractive materials and their applications: Topics in Applied Physics, Springer, 1989.

URL https://books.google.com.br/books?id=TD_xAAAAMAAJ

[2] S. G. Odoulov, K. V. Shcherbin, A. N. Shumeljuk, Photorefractive recording in BTO in the near infrared, J. Opt. Soc. Am. B 11 (9) (1994) 1780-1785. doi:10.1364/JOSAB.11.001780.

URL http://josab.osa.org/abstract.cfm?URI=josab-11-9-1780

[3] P. dos Santos, J. Frejlich, J. Carvalho, Direct near infrared photorefractive recording and pre-exposure controlled hole-electron competition with enhanced recording in undoped $\mathrm{Bi}_{12} \mathrm{TiO}_{20}$, Applied Physics B 81 (5) (2005) 651-655. doi:10.1007/s00340-005-1935-x.

URL https://doi.org/10.1007/s00340-005-1935-x

[4] A. L. Moura, A. A. Canabarro, W. C. Soares, E. de Lima, J. F. Carvalho, P. V. dos Santos, Experimental determination of effective electro-optic coefficient and electric screening field factor in the electrically induced birefringent $\mathrm{Bi}_{12} \mathrm{TiO}_{20}$ crystal by using an oblique incidence setup, Optics Communications 295 (Supplement C) (2013) 197 - 202. doi:https://doi.org/10.1016/j.optcom.2012.12.085.

URL http://www.sciencedirect.com/science/article/pii/S0030401813000163

[5] J. P. Huignard, F. Micheron, High-sensitivity read-write volume holographic storage in $\mathrm{Bi}_{12} \mathrm{SiO}_{20}$ and $\mathrm{Bi}_{12} \mathrm{GeO}_{20}$ crystals, Applied Physics Letters 29 (9) (1976) 591-593. doi:10.1063/1.89153.

URL http://dx.doi.org/10.1063/1.89153

[6] N. V. Kukhtarev, V. B. Markov, S. G. Odulov, M. S. Soskin, V. L. Vinetskii, Holographic storage in electrooptic crystals. i. steady state, Ferroelectrics 22 (1) (1978) 949-960. doi:10.1080/00150197908239450.

URL http://dx.doi.org/10.1080/00150197908239450

[7] A. Marrakchi, R. V. Johnson, A. R. Tanguay, Polarization properties of photorefractive diffraction in electrooptic and optically active sillenite crystals (bragg regime), J. Opt. Soc. Am. B 3 (2) (1986) 321-336. doi:10.1364/JOSAB.3.000321.

URL http://josab.osa.org/abstract.cfm?URI=josab-3-2-321

[8] J. Huignard, A. Marrakchi, Coherent signal beam amplification in two-wave mixing experiments with photorefractive $\quad \mathrm{Bi}_{12} \mathrm{SiO}_{20} \quad$ crystals, Optics $\quad$ Communications 38 (4) (1981) 249 - 254 . doi:https://doi.org/10.1016/0030-4018(81)90392-8.

URL http://www.sciencedirect.com/science/article/pii/ 0030401881903928

[9] P. Refregier, L. Solymar, H. Rajbenbach, J. P. Huignard, Two-beam coupling in photorefractive $\mathrm{Bi}_{12} \mathrm{SiO}_{20}$ crystals with moving grating: Theory and experiments, Journal of Applied Physics 58 (1) (1985) 45-57. arXiv:http://dx.doi.org/10.1063/1.335646,doi:10.1063/1.335646. 
URL http://dx.doi.org/10.1063/1.335646

[10] S. Stepanov, M. Petrov, Efficient unstationary holographic recording in photorefractive crystals under an external alternating electric field, Optics Communications 53 (5) (1985) 292 - 295. doi:https://doi.org/10.1016/0030-4018(85)90265-2.

URL http://www.sciencedirect.com/science/article/pii/0030401885902652

[11] A. A. Freschi, A. C. C. Telles, J. Frejlich, D. A. Donatti, Oscillating holograms recorded in photorefractive crystals by a frequency detuned feedback loop, Journal of Applied Physics 105 (2) (2009) 023109. arXiv:http://dx.doi.org/10.1063/1.3072684, doi:10.1063/1.3072684.

URL http://dx.doi.org/10.1063/1.3072684

[12] J. Frejlich, Photorefractive materials: fundamental concepts, holographic recording and materials characterization, Wiley-Interscience, 2007.

URL https://books.google.com.br/books?id=4axRAAAAMAAJ

[13] J. Frejlich, R. Montenegro, N. R. Inocente-Junior, P. V. dos Santos, J. C. Launay, C. Longeaud, and J. F. Carvalho, Phenomenological characterization of photoactive centers in $\mathrm{Bi}_{12} \mathrm{TiO}_{20}$ crystals, J. Appl. Phys. 101 (2007) 043101-1 - 043101-12. doi:10.1063/1.2434009.

URL https://doi.org/10.1063/1.2434009

[14] P. Günter, J. Huignard, Photorefractive Materials and Their Applications 1: Basic Effects, Springer Series in Optical Sciences, Springer New York, 2005.

URL https://books.google.com.br/books?id=we9AAAAACAAJ

[15] M. B. Klein, S. W. McCahon, T. F. Boggess, G. C. Valley, High-accuracy, high-reflectivity phase conjugation at $1.06 \mu \mathrm{m}$ by four-wave mixing in photorefractive gallium arsenide, J. Opt. Soc. Am. B 5 (12) (1988) 2467-2472. doi:10.1364/JOSAB.5.002467.

URL http://josab.osa.org/abstract.cfm?URI=josab-5-12-2467

[16] A. Grunnet-Jepsen, I. Aubrecht, L. Solymar, Investigation of the internal field in photorefractive materials and measurement of the effective electro-optic coefficient, J. Opt. Soc. Am. B 12 (5) (1995) 921929. doi:10.1364/JOSAB.12.000921.

URL http://josab.osa.org/abstract.cfm?URI=josab-12-5-921

[17] A. A. Kamshilin, E. Raita, A. V. Khomenko, Intensity redistribution in a thin photorefractive crystal caused by strong fanning effect and internal reflections, J. Opt. Soc. Am. B 13 (11) (1996) 2536-2543. doi:10.1364/JOSAB.13.002536.

URL http://josab.osa.org/abstract.cfm?URI=josab-13-11-2536

[18] M. B. Klein, G. C. Valley, Beam coupling in $\mathrm{BaTiO}_{3}$ at 442 nm, Journal of Applied Physics 57 (11) (1985) 4901-4905. arXiv:http://dx.doi.org/10.1063/1.335313, doi:10.1063/1.335313.

URL http://dx.doi.org/10.1063/1.335313 